\title{
Poiseuille channel flow by adding and doubling
}

Barry D. Ganapol

Citation: 1786, 070009 (2016); doi: 10.1063/1.4967585

View online: http://dx.doi.org/10.1063/1.4967585

View Table of Contents: http://aip.scitation.org/toc/apc/1786/1

Published by the American Institute of Physics 


\title{
Poiseuille Channel Flow by Adding and Doubling
}

\author{
Barry D. Ganapol \\ Department of Aerospace and Mechanical Engineering \\ University of Arizona \\ Ganapol@cowboy.ame.arizona.edu
}

\begin{abstract}
The flow of a rarefied gas between two plates of a micro channel, commonly called Poiseuille flow, has been extensively studied in the BGK approximation. In particular, the Analytical Discrete Ordinates (ADO) method determines the velocity profile and flow rate efficiently and accurately. Here, an equally efficient and precise solution is presented using the method of doubling, which however is arguably a numerically simpler and a more natural approach avoiding eigenvalues and eigenvectors. Highly accurate benchmark results for Poiseuille flow are reported for use as a benchmark.
\end{abstract}

\section{INTRODUCTION}

Poiseuille flow is one of, if not, the simplest of all RGD flows. It is molecular flow down a pipe or between plates as shown in Fig. 1. Here the flow is in the $z$-direction in a transversely infinite channel of height $d$. The flowing

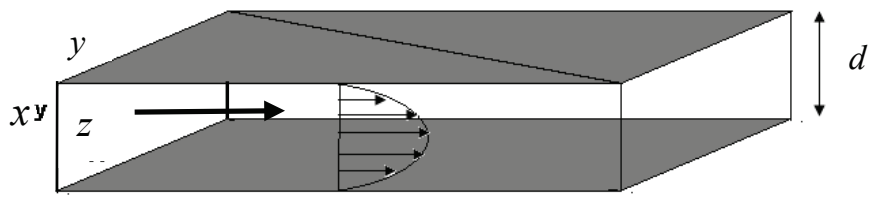

FIGURE 1. Poiseuille micro channel flow.

molecules slip along the upper and lower channel plates to varying degrees. The accommodation coefficient $\alpha$ is a flow parameter indicating the strength of slip at the wall. The fundamental assumption for Poiseuille flow is a long wide channel with laminar flow down the channel as described by the BGK scattering model approximation. The challenge to Poiseuille flow is to determine the molecular distribution in the $x$-direction perpendicular to the flow and the corresponding flow rate.

The most straightforward treatment for Poiseuille flow is to linearize the density distribution function as

$$
f(x, c)=f_{0}(x, c)[1+h(x, c)]
$$

where $f_{0}(x, \boldsymbol{c})$ is a Maxwellian and $h(x, \boldsymbol{c})$ is a perturbation from the Maxwellian to be determined from the linearized Boltzmann equation

$$
k c_{z}+c_{x} \frac{\partial}{\partial x} h(x, c)=\boldsymbol{L} h(x, c)
$$

where $\boldsymbol{L}$ is the BGK operator ${ }^{1}$ and 


$$
k \equiv \frac{1}{P} \frac{\partial P}{\partial x}
$$

is the pressure gradient driving the flow. The analysis continues with model reduction through integration and the definition of the moment

$$
Z\left(x, c_{x}\right)=\pi^{-1} \int_{-\infty}^{\infty} \int_{-\infty}^{\infty} e^{-\left(c_{y}^{2}+c_{z}^{2}\right)} c_{z} h\left(x, c_{x}, c_{y}, c_{z}\right) \mathrm{d} c_{y} \mathrm{~d} c_{z}
$$

leading to

$$
\frac{1}{2} k \theta+\theta c_{x} \frac{\partial}{\partial x} Z\left(x, c_{x}\right)+Z\left(x, c_{x}\right)=\pi^{-1 / 2} \int_{-\infty}^{\infty} e^{-u^{2}} Z(x, u) \mathrm{d} u
$$

for $x \in(-d / 2, d / 2)$ where $\theta$ is the mean free path of molecular scattering. The boundary conditions are

$$
\begin{aligned}
& Z\left(-d / 2, c_{x}\right)=(1-\alpha) Z\left(-d / 2,-c_{x}\right) \\
& Z\left(d / 2,-c_{x}\right)=(1-\alpha) Z\left(d / 2, c_{x}\right)
\end{aligned}
$$

where $\alpha$ is the accommodation coefficient specifying the degree of slip satisfying $0 \leq \alpha \leq 1$. On further change of variables $\tau=x / \theta, \mu=c_{x}$, we find

$$
\frac{1}{2} k \theta+\mu \frac{\partial}{\partial \tau} Z(\tau, \mu)+Z(\tau, \mu)=\pi^{-1 / 2} \int_{-\infty}^{\infty} e^{-u^{2}} Z(\tau, u) \mathrm{d} u
$$

with $\tau \in(-\delta / 2, \delta / 2), \mu \in(-\infty, \infty)$ and boundary conditions

$$
\begin{aligned}
& Z(-\delta / 2, \mu)=(1-\alpha) Z(-\delta / 2,-\mu) \\
& Z(\delta / 2,-\mu)=(1-\alpha) Z(\delta / 2, \mu)
\end{aligned}
$$

where

$$
\delta \equiv \frac{d}{\theta} \equiv 2 a
$$

is the inverse of the Knudsen number.

The equation we finally solve comes with the substitution

$$
Z(\tau, \mu)=\frac{1}{2} k \theta\left[\tau^{2}-2 \tau \mu+2 \mu^{2}-a^{2}-2 Y(\tau, \mu)\right]
$$

to give

$$
\left[\mu \frac{\partial}{\partial \tau}+1\right] Y(\tau, \mu)=\int_{-\infty}^{\infty} d \mu^{\prime} \Psi\left(\mu^{\prime}\right) Y\left(\tau, \mu^{\prime}\right)
$$

where $\mu \in(-\infty, \infty), \tau \in(0, a)$, and the boundary conditions are 


$$
\begin{aligned}
& Y(0, \mu)=Y(0,-\mu) \\
& Y(a,-\mu)=(1-\alpha) Y(a, \mu)+g(\mu) \\
& g(\mu) \equiv \alpha \mu^{2}+a(2-\alpha) \mu
\end{aligned}
$$

with $\mu \in[0, \infty)$ and $\Psi(\mu) \equiv \pi^{-1 / 2} e^{-\mu^{2}}$.

\section{THEORY OF ADDING AND DOUBLING}

There have been several different approaches to solve Eqs(6) including the variational method of Cercignani and Loranzani ${ }^{1}$, Elementary solutions by Loyalka ${ }^{2}$, et. al., Fourier transform by Boffi ${ }^{3}$, et. al. and the FN method of Siewert, Garcia and Barichello ${ }^{4}$. All these approaches involve relatively intricate mathematical constructions as well as elaborate numerical methods. What is proposed here is more fundamental and natural, yet provides as accurate, or more accurate results than the precise methods existing today.

\section{Discrete Ordinates Balance Equation}

In this section, we derive the discrete ordinates balance equation by specifying a more familiar form of $\mathrm{Eq}(6 \mathrm{a})$ through the variable $u, 0 \leq u \leq 1$, and introducing the result into $\mathrm{Eq}(6 \mathrm{a})$

$$
\left[\mu(u) \frac{\partial}{\partial \tau}+1\right] Y(\tau, \mu(u))=\int_{-\infty}^{\infty} d \mu^{\prime} \Psi\left(\mu\left(u^{\prime}\right)\right) Y\left(\tau, \mu\left(u^{\prime}\right)\right),
$$

with dependence on $u$ and $u^{\prime}$ made explicit. One can then partition the integral into its half range integrations

$$
\int_{-\infty}^{\infty} d \mu^{\prime} \Psi\left(\mu^{\prime}\right) Y\left(\tau, \mu^{\prime}\right)=\int_{0}^{1} d u^{\prime} \frac{d \mu^{\prime}\left(u^{\prime}\right)}{d u^{\prime}} \Psi\left(\mu^{\prime}\left(u^{\prime}\right)\right)\left[\begin{array}{c}
Y\left(\tau, \mu^{\prime}\left(u^{\prime}\right)\right)+ \\
+Y\left(\tau,-\mu^{\prime}\left(u^{\prime}\right)\right)
\end{array}\right] .
$$

Therefore, for $\mu \in(0, \infty)^{4}$, and

$$
u \equiv \frac{1}{1+\mu}
$$

we have

$$
\int_{-\infty}^{\infty} d \mu^{\prime} \Psi\left(\mu^{\prime}\right) Y\left(\tau, \mu^{\prime}\right)=\int_{0}^{1} d u^{\prime} u^{\prime-2} \Psi\left(\mu^{\prime}\left(u^{\prime}\right)\right)\left[\begin{array}{c}
Y\left(\tau, \mu^{\prime}\left(u^{\prime}\right)\right)+ \\
+Y\left(\tau,-\mu^{\prime}\left(u^{\prime}\right)\right)
\end{array}\right] .
$$

When we approximate the half-range integration by a Gauss quadrature of order $N$, there results

$$
\int_{-\infty}^{\infty} d \mu^{\prime} \psi\left(\mu^{\prime}\right) Y\left(\tau, \mu^{\prime}\right)=\sum_{m^{\prime}=0}^{N} \omega_{m^{\prime}} \Psi_{m^{\prime}}\left[Y_{m^{\prime}}^{+}(\tau)+Y_{m^{\prime}}^{-}(\tau)\right]+E_{N}(\tau),
$$

where

$$
Y\left(\tau, \pm \mu_{m}\right)=Y_{m}^{ \pm}(\tau)+\varepsilon_{m}(\tau)
$$


In the above expressions, $E_{N}(\tau)$ is the quadrature error and $\varepsilon_{m}(\tau)$ is the local error in $Y$.

The abscissae come from zeros of the Legendre polynomial of degree $N$

$$
\begin{aligned}
& P_{N}\left(x_{m}\right)=0, x_{m} \equiv 2 u_{m}-1, m=1, \ldots, N \\
& u_{m}=\frac{1}{2}\left(1+x_{m}\right)
\end{aligned}
$$

giving the half-range ordinates for $\mu \in(-\infty, \infty)$

$$
\mu_{m}=\frac{u_{m}}{1-u_{m}}, \mu_{N+m}=-u_{m}
$$

and weights

$$
\omega_{m}=\omega_{N+m} \equiv \frac{2}{(N+1)^{2}\left[P_{N+1}\left(u_{m}\right)\right]^{2}} \frac{1}{u_{m}{ }^{2}} .
$$

Introducing $\mathrm{Eq}(10 \mathrm{a})$ into $\mathrm{Eq}(7)$, evaluating at $\pm \mu_{m}$ and dropping the error term gives the following the discrete ordinates approximation in the two half-ranges:

$$
\begin{aligned}
& {\left[\mu_{m} \frac{\partial}{\partial \tau}+1\right] Y_{m}^{+}(\tau)=\sum_{m^{\prime}=0}^{N} \omega_{m^{\prime}} \Psi_{m^{\prime}}\left[Y_{m^{\prime}}^{+}(\tau)+Y_{m^{\prime}}^{-}(\tau)\right]} \\
& {\left[-\mu_{m} \frac{\partial}{\partial \tau}+1\right] Y_{m}^{-}(\tau)=\sum_{m^{\prime}=0}^{N} \omega_{m^{\prime}} \Psi_{m^{\prime}}\left[Y_{m^{\prime}}^{+}(\tau)+Y_{m^{\prime}}^{-}(\tau)\right] .}
\end{aligned}
$$

A more convenient form is found if one defines the vectors in the positive (+) and negative (-) half-ranges

$$
Y^{ \pm}(\tau) \equiv\left[\begin{array}{llll}
Y_{1}^{ \pm}(\tau) & Y_{2}^{ \pm}(\tau) \quad \ldots & Y_{N}^{ \pm}(\tau)
\end{array}\right]^{T}
$$

for which $\operatorname{Eqs}(12)$ becomes

$$
\begin{aligned}
& {\left[\frac{d}{d \tau}+\boldsymbol{M}^{-1}(\boldsymbol{I}-\boldsymbol{C})\right] \boldsymbol{Y}^{+}(\tau)=\boldsymbol{M}^{-1} \boldsymbol{C} \boldsymbol{Y}^{-}(\tau) } \\
{\left[-\frac{d}{d \tau}+\boldsymbol{M}^{-1}(\boldsymbol{I}-\boldsymbol{C})\right] \boldsymbol{Y}^{-}(\tau)=\boldsymbol{M}^{-1} \boldsymbol{C} \boldsymbol{Y}^{+}(\tau) } & \\
\boldsymbol{\Psi} & \equiv \operatorname{diag}\left\{\Psi_{m} ; j=1, \ldots, J\right\} \\
\boldsymbol{W} & \equiv \operatorname{diag}\left\{\omega_{m} ; j=1, \ldots, J\right\} \\
\boldsymbol{M} & \equiv \operatorname{diag}\left\{\mu_{m} ; j=1, \ldots, J\right\} \\
\boldsymbol{C} & \equiv \boldsymbol{W} \boldsymbol{\Psi} .
\end{aligned}
$$

From $\mathrm{Eq}(6 \mathrm{~b}, \mathrm{c}, \mathrm{d})$, the boundary conditions are 


$$
\begin{aligned}
& Y_{N+m}(0)=Y_{m}(0) \\
& Y_{m}(a)=(1-\alpha) Y_{N+m}(a)+g_{N+m} \\
& g_{N+m} \equiv \alpha \mu_{N+m}^{2}+a(2-\alpha) \mu_{N+m}^{2} .
\end{aligned}
$$

\section{Solution by Adding and Doubling}

In this section, we will find the solution to Eqs(14) by adding and doubling.

If

$$
\boldsymbol{\phi}(\tau) \equiv\left[\begin{array}{ll}
\boldsymbol{Y}^{T+}(\tau) & \boldsymbol{Y}^{T-}(\tau)
\end{array}\right]^{T}
$$

then

$$
\frac{d \phi(\tau)}{d \tau}+\boldsymbol{A} \phi(\tau)=0,
$$

with

$$
\boldsymbol{A}=\left[\begin{array}{cc}
\boldsymbol{M}^{-1}(\boldsymbol{I}-\boldsymbol{C}) & -\boldsymbol{M}^{-1} \boldsymbol{C} \\
\boldsymbol{M}^{-1} \boldsymbol{C} & -\boldsymbol{M}^{-1}(\boldsymbol{I}-\boldsymbol{C})
\end{array}\right]
$$

where $\boldsymbol{I}$ is the (size $N$ ) identity matrix.

Assuming a uniform interval discretization $h$, the exact solution to $\mathrm{Eq}(15 \mathrm{~b})$ over the interval $\left[\tau_{j}, \tau_{j+1}\right]$ is

$$
\phi_{j+1}=e^{-\boldsymbol{A} h} \phi_{j} .
$$

Certainly, one can evaluate the matrix exponential by finding the eigenvalues and eigenvectors of $A$, as is effectively done by the $\mathrm{ADO}$ method $^{4}$, but this is unnecessary for the most convenient numerical result. All that is required is the Padé approximant

with

$$
e^{-\boldsymbol{A} h} \simeq \boldsymbol{P}^{*-1} \boldsymbol{P}
$$

$$
\begin{aligned}
& \boldsymbol{P}^{*} \equiv[\boldsymbol{I}+h \boldsymbol{A} / 2]^{-1} \\
& \boldsymbol{P} \equiv[\boldsymbol{I}-h \boldsymbol{A} / 2]
\end{aligned}
$$

to give the solution over the interval

$$
\left[\begin{array}{c}
\boldsymbol{Y}_{j}^{-} \\
\boldsymbol{Y}_{j+1}^{+}
\end{array}\right]=\boldsymbol{R}\left[\begin{array}{c}
\boldsymbol{Y}_{j+1}^{-} \\
\boldsymbol{Y}_{j}^{+}
\end{array}\right]
$$

where, after some algebra, the interval response matrix $\boldsymbol{R}$ is 


$$
\boldsymbol{R}=\left[\begin{array}{cc}
\alpha_{+}-\beta & \alpha_{+}-\beta \\
\alpha_{+}+\beta & -\left(\alpha_{+}+\beta\right)
\end{array}\right]^{-1}\left[\begin{array}{cc}
\alpha_{-}+\beta & \alpha_{-}+\beta \\
\alpha_{-}-\beta & -\left(\alpha_{-}-\beta\right)
\end{array}\right]
$$

with

$$
\begin{aligned}
& \boldsymbol{\alpha}_{+} \equiv \boldsymbol{I}+\frac{h}{2} \boldsymbol{M}^{-1}(\boldsymbol{I}-\boldsymbol{C}), \boldsymbol{\beta} \equiv \frac{h}{2} \boldsymbol{M}^{-1} \boldsymbol{C} \\
& \boldsymbol{\alpha}_{-} \equiv \boldsymbol{I}-\frac{h}{2} \boldsymbol{M}^{-1}(\boldsymbol{I}-\boldsymbol{C}) .
\end{aligned}
$$

It is important to note that the RHS vector in $\mathrm{Eq}(18 \mathrm{a})$ is what comes into the interval from either side, and the LHS vector is what leaves, or the response.

In addition, one can write

$$
\left\{\begin{array}{l}
\boldsymbol{T}_{n} \\
\boldsymbol{R}_{f}
\end{array}\right\}=\frac{1}{2}\left[\left(\boldsymbol{\alpha}_{+}-\boldsymbol{\beta}_{+}\right)^{-1}\left(\boldsymbol{\alpha}_{-}+\boldsymbol{\beta}_{-}\right) \pm\left(\boldsymbol{\alpha}_{+}+\boldsymbol{\beta}_{+}\right)^{-1}\left(\boldsymbol{\alpha}_{-}-\boldsymbol{\beta}_{-}\right)\right]
$$

and

$$
\boldsymbol{R}=\left[\begin{array}{cc}
\boldsymbol{T}_{n} & \boldsymbol{R}_{f} \\
\boldsymbol{R}_{f} & \boldsymbol{T}_{n}
\end{array}\right]
$$

where $\boldsymbol{R}_{\boldsymbol{n}}$ and $\boldsymbol{T}_{\boldsymbol{n}}$ are the interval reflectance and transmittance, which are naturally symmetric.

It is now possible to add two homogeneous media together to find a composite response. Figure 2

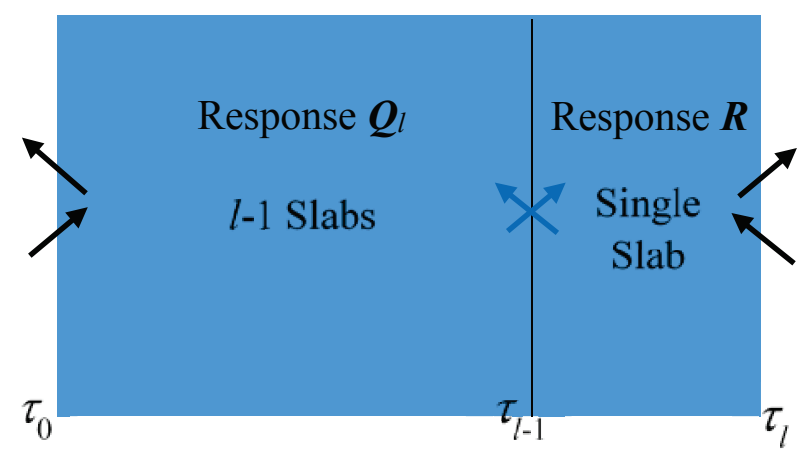

FIGURE 2. Concatenation of two homogeneous media.

shows the combination of two slabs of different responses. By eliminating the densities at the interface, one finds, with some algebra,

$$
\left[\begin{array}{c}
\boldsymbol{Y}_{0}^{-} \\
\boldsymbol{Y}_{l}^{+}
\end{array}\right]=\boldsymbol{Q}_{l}\left[\begin{array}{l}
\boldsymbol{Y}_{l}^{-} \\
\boldsymbol{Y}_{0}^{+}
\end{array}\right]
$$

where 


$$
\begin{gathered}
\boldsymbol{Q}_{l}=\left[\begin{array}{cc}
\mathbf{0} & \boldsymbol{Q}_{l-1,1} \\
\boldsymbol{T}_{n} & \mathbf{0}
\end{array}\right] \boldsymbol{U}_{l-1}+\left[\begin{array}{cc}
\mathbf{0} & \boldsymbol{Q}_{l-1,2} \\
\boldsymbol{R}_{f} & \mathbf{0}
\end{array}\right] \\
\boldsymbol{U}_{l-1}=\left[\begin{array}{cc}
\boldsymbol{Q}_{l-1,2}\left[\boldsymbol{I}-\boldsymbol{R}_{f} \boldsymbol{Q}_{l-1,2}\right]^{-1} \boldsymbol{T}_{n} & \boldsymbol{Q}_{l-1,1}+\boldsymbol{Q}_{l-1,2}\left[\boldsymbol{I}-\boldsymbol{R}_{f} \boldsymbol{Q}_{l-1,2}\right]^{-1} \boldsymbol{R}_{f} \boldsymbol{Q}_{l-1,1} \\
{\left[\boldsymbol{I}-\boldsymbol{R}_{f} \boldsymbol{Q}_{l-1,2}\right]^{-1} \boldsymbol{T}_{n}} & {\left[\boldsymbol{I}-\boldsymbol{R}_{f} \boldsymbol{Q}_{l-1,2}\right]^{-1} \boldsymbol{R}_{f} \boldsymbol{Q}_{l-1,1}}
\end{array}\right]
\end{gathered}
$$

The solution procedure is as follows:

+ Divide the channel into $2^{n}$ uniform intervals and call the intervals $l=1,2, \ldots, n$.

+ Find the response $Q_{1}$ for interval $l=1$.

+ Combine two such intervals using $\mathrm{Eq}(20 \mathrm{~b})$ and (20c) to form $Q_{2}$.

+ Now combine the last 2 intervals with the next 2 intervals and continue doubling to the $l$ th interval to form the response $Q_{2}{ }^{l}$.

+ Eventually cover the entire channel to give the channel response $Q(n)=Q_{2^{n}}$.

Now $\mathrm{Eq}(20 \mathrm{a})$ represents the solution over the channel

$$
\left[\begin{array}{c}
\boldsymbol{Y}_{0}^{-} \\
\boldsymbol{Y}_{n}^{+}
\end{array}\right]=\boldsymbol{Q}_{n}\left[\begin{array}{c}
\boldsymbol{Y}_{n}^{-} \\
\boldsymbol{Y}_{0}^{+}
\end{array}\right]
$$

where, for convenience

$$
\boldsymbol{Q}_{n} \equiv \boldsymbol{Q}(n)
$$

and from Eqs(14e,f,g)

$$
\left[\begin{array}{l}
\boldsymbol{Y}_{n}^{-} \\
\boldsymbol{Y}_{0}^{+}
\end{array}\right]=\left[\begin{array}{c}
(1-\alpha) \boldsymbol{Y}_{n}^{+}+\boldsymbol{g}^{+} \\
\boldsymbol{Y}_{0}^{-}
\end{array}\right]=\left[\begin{array}{cc}
0 & (1-\alpha) \boldsymbol{I} \\
\boldsymbol{I} & 0
\end{array}\right]\left[\begin{array}{l}
\boldsymbol{Y}_{0}^{-} \\
\boldsymbol{Y}_{n}^{+}
\end{array}\right]+\left[\begin{array}{l}
\boldsymbol{g}^{+} \\
0
\end{array}\right] .
$$

When we introduce $\mathrm{Eq}(21 \mathrm{c})$ into $\mathrm{Eq}(21 \mathrm{a})$ and solve for the exiting vector, we find the solution

$$
\left[\begin{array}{c}
\boldsymbol{Y}_{0}^{-} \\
\boldsymbol{Y}_{n}^{+}
\end{array}\right]=\left[\boldsymbol{I}-\boldsymbol{Q}_{n}\left[\begin{array}{cc}
\mathbf{0} & (1-\alpha) \boldsymbol{I} \\
\boldsymbol{I} & \mathbf{0}
\end{array}\right]\right]^{-1}\left[\begin{array}{c}
\boldsymbol{g}^{+} \\
\mathbf{0}
\end{array}\right]
$$

where $\boldsymbol{g}^{+} \equiv \boldsymbol{M}[\alpha \boldsymbol{M}+a(2-\alpha) \boldsymbol{I}] \mathbf{1}$.

\section{Numerical Implementation and Demonstration}

For this presentation, because of space limitation, it will suffice to determine only the channel flow rate to high precision.

After some manipulations, one can show the flow rate for a channel of width $a$ is

$$
\begin{aligned}
& Q(a, \alpha ; N, n)=B(a, \alpha)+\frac{2}{a^{2} \sqrt{\pi}}[\Psi \boldsymbol{W}]^{T}\left[\alpha \boldsymbol{M}^{3}+a(2-\alpha) \boldsymbol{M}^{2}\right] \boldsymbol{Y}_{n}^{+} \\
& B(a, \alpha) \equiv \frac{a}{3}-\frac{1}{2 a}+\frac{\alpha}{a}\left[\frac{3}{4}-\frac{2}{a \sqrt{\pi}}\right]-(2-\alpha)\left[\frac{3}{4 a}-\frac{1}{\sqrt{\pi}}\right] .
\end{aligned}
$$


Note the dependence on the quadrature order $N$ and channel discretization are explicitly included, since at this point, we have the solution as a function of the discretizations. For this reason, there is an opportunity for convergence acceleration $^{6}$, which enables a high order evaluation of the following double limit:

$$
Q(a, \alpha)=\lim _{N \rightarrow \infty} \lim _{n \rightarrow \infty} Q(a, \alpha ; N, n)
$$

The idea is to use Wynn-epsilon in $N$ and Richardsons convergence acceleration in $n$ to form a surrogate sequence $\hat{Q}(a, \alpha ; N, n)$, which, generally, converges faster to the limit of $\mathrm{Eq}(24)$ than the original sequence.

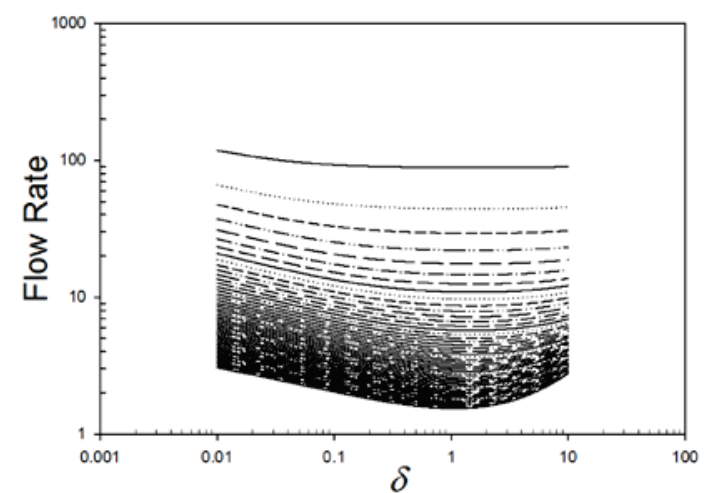

FIGURE 3. Flow variation with inverse Knudsen number.
The surrogate sequence will usually satisfy

$$
\frac{Q(a, \alpha)-\hat{Q}(a, \alpha ; N, n)}{Q(a, \alpha)-Q(a, \alpha ; N, n)} \rightarrow 0
$$

indicating the numerator converges faster than the original sequence. Figure 3 shows the characteristic behavior of $Q$ with decreasing Knusdsen number ${ }^{1}$ to fully developed flow.

Finally, Table 1 gives highly precise benchmark values to be used as a standard. The values are in complete agreement with those of Ref. 4 with two additional digits.

Table 1. Benchmark values for flow rate.

\begin{tabular}{cccccc}
\hline $\boldsymbol{\tau} \boldsymbol{\alpha} \boldsymbol{\alpha}$ & $\mathbf{0 . 5}$ & $\mathbf{0 . 8}$ & $\mathbf{0 . 8 8}$ & $\mathbf{0 . 9 6}$ & $\mathbf{1 . 0 0}$ \\
\hline $5.000 \mathrm{E}-02$ & $5.2232964 \mathrm{E}+00$ & $3.0897113 \mathrm{E}+00$ & $2.7383403 \mathrm{E}+00$ & $2.4373544 \mathrm{E}+00$ & $2.3022564 \mathrm{E}+00$ \\
$1.000 \mathrm{E}-01$ & $4.5564062 \mathrm{E}+00$ & $2.7077408 \mathrm{E}+00$ & $2.4060457 \mathrm{E}+00$ & $2.1482414 \mathrm{E}+00$ & $2.0327143 \mathrm{E}+00$ \\
$3.000 \mathrm{E}-01$ & $3.7784723 \mathrm{E}+00$ & $2.2447708 \mathrm{E}+00$ & $2.0010675 \mathrm{E}+00$ & $1.7945088 \mathrm{E}+00$ & $1.7024740 \mathrm{E}+00$ \\
$5.000 \mathrm{E}-01$ & $3.5443709 \mathrm{E}+00$ & $2.1022657 \mathrm{E}+00$ & $1.8766202 \mathrm{E}+00$ & $1.6863424 \mathrm{E}+00$ & $1.6018742 \mathrm{E}+00$ \\
$7.000 \mathrm{E}-01$ & $3.4376693 \mathrm{E}+00$ & $2.0387670 \mathrm{E}+00$ & $1.8220109 \mathrm{E}+00$ & $1.6398495 \mathrm{E}+00$ & $1.5591860 \mathrm{E}+00$ \\
$9.000 \mathrm{E}-01$ & $3.3838869 \mathrm{E}+00$ & $2.0092408 \mathrm{E}+00$ & $1.7976360 \mathrm{E}+00$ & $1.6202230 \mathrm{E}+00$ & $1.5417996 \mathrm{E}+00$ \\
$1.000 \mathrm{E}+00$ & $3.3682182 \mathrm{E}+00$ & $2.0018669 \mathrm{E}+00$ & $1.7920590 \mathrm{E}+00$ & $1.6163124 \mathrm{E}+00$ & $1.5386785 \mathrm{E}+00$ \\
$2.000 \mathrm{E}+00$ & $3.3765738 \mathrm{E}+00$ & $2.0413852 \mathrm{E}+00$ & $1.8385632 \mathrm{E}+00$ & $1.6693655 \mathrm{E}+00$ & $1.5948569 \mathrm{E}+00$ \\
$5.000 \mathrm{E}+00$ & $3.7744018 \mathrm{E}+00$ & $2.4382339 \mathrm{E}+00$ & $2.2350591 \mathrm{E}+00$ & $2.0654781 \mathrm{E}+00$ & $1.9907674 \mathrm{E}+00$ \\
$7.000 \mathrm{E}+00$ & $4.0881078 \mathrm{E}+00$ & $2.7461124 \mathrm{E}+00$ & $2.5414362 \mathrm{E}+00$ & $2.3703751 \mathrm{E}+00$ & $2.2949322 \mathrm{E}+00$ \\
$9.000 \mathrm{E}+00$ & $4.4101902 \mathrm{E}+00$ & $3.0634644 \mathrm{E}+00$ & $2.8575645 \mathrm{E}+00$ & $2.6852950 \mathrm{E}+00$ & $2.6092536 \mathrm{E}+00$ \\
\hline
\end{tabular}

\section{CONCLUSION}

The adding and doubling method is applied for the first time in RGD. The novelty of the approach is that no eigenvalues or eigenvectors are required. We only require quadrature and a Padé approximant. In spite of the method's simplicity, one finds highly precise flow rates.

\section{REFERENCES}

1. C. Cercignani and S. Lorenzani, A Variational Solution of the Linearized Boltzmann Equation for Plane Poiseuille Flow, AIP Conf. Proc. 1084, 63 (2008); 10.1063/1.3076555.

2. S. K. Loyalka, N. Petrellis and T. S. Storvick, Z. Angew. Math. Phys. 30, 514, (1979.

3. V. Boffi, L. DeSocio,G. Gaffuri and C.Pescatore, Meccanica 11 (1976).

4. L. B. Barichello and C. E. Siewert, A discrete-ordinates solution for Poiseuille flow in a plane channel, Z. angew. Math. Phys. 50 pp. 972-981, (1999).

5. C.E. Siewert, R.D.M. Garcia and P. Grandjean, J. Math. Phys. 21, 2760 (1980).

6. A. Sidi, Practical Extrapolations Methods, Cambridge University Press, Cambridge, (2003). 